Service social

\title{
Intervention de groupe et approche féministe en santé mentale
}

\section{Michèle Brousseau}

Volume 39, numéro 1, 1990

Le groupe ici et ailleurs

URI : https://id.erudit.org/iderudit/706459ar

DOI : https://doi.org/10.7202/706459ar

Aller au sommaire du numéro

Éditeur(s)

École de service social de l'Université Laval

ISSN

1708-1734 (numérique)

Découvrir la revue

Citer cet article

Brousseau, M. (1990). Intervention de groupe et approche féministe en santé mentale. Service social, 39(1), 97-113. https://doi.org/10.7202/706459ar

\section{Résumé de l'article}

Les femmes sont les principales utilisatrices des services psychiatriques au Québec, et tant les approches traditionnelles que les stéréotypes sexistes sont l'objet de critiques. Une aide offerte en groupe et avec une approche féministe peut-elle répondre aux besoins de ces femmes?

L'article présente un programme développé en réponse à cette interrogation à partir d'une recension des publications sur le thème de la santé mentale des femmes dans une perspective féministe et de groupe. On y donne les résultats d'une étude évaluant les effets d'un programme de groupe avec approche féministe sur l'estime de soi, l'affirmation de soi et l'état dépressif des participantes suivies à la clinique externe d'un hôpital général. Le cadre de la recherche et la méthodologie sont aussi abordés brièvement. L'article se termine par une discussion des résultats, leurs limites et les implications pour la recherche. 
Michèle Brousseau, travailleuse sociale au Centre de services sociaux de Québec.

\title{
Intervention de groupe et approche féministe en santé mentale
}

\author{
Michèle Brousseau
}

L'approche féministe en intervention de groupe peut-elle être utile à des femmes qui viennent consulter en psychiatrie, qu'elles soient les habituées plutôt passives des services ou qu'elles en soient à leur premier appel à l'aide? Ainsi peut se résumer la question de départ qui a orienté la recherche dont nous faisons ici état. En effet, malgré les récents développements de l'intervention féministe et son utilisation en service social des groupes, tant au niveau de l'enseignement que de la pratique, et la pertinence d'évaluer les effets de nos interventions, il n'existe pas, à notre connaissance, de recherche québécoise sur le sujet.

Cette recherche évaluative a été menée dans le cadre d'études de maîtrise en service social à l'Université Laval auprès de clientes de la clinique externe de psychiatrie d'un hôpital général. Le présent article s'inspire du contenu de ce mémoire de maîtrise' ${ }^{1}$.

\section{Femmes et santé mentale : une perspective féministe et de groupe}

\section{Profil de la clientèle}

Les femmes représentent les deux tiers des usagers des services psychiatriques, surtout les femmes mariées et à partir de la trentaine; elles présentent des problèmes différents de ceux des hommes, surtout la dépression et l'anxiété (Chesler, 1979; DeKoninck et al., 1981; Lebeau et al., 1979). Elles reçoivent aussi des services différents : plus de médicaments (Cooperstock, 1980; Lebeau et al., 
1981; DeKoninck et al., 1981), plus d'électrochocs (Guyon et al., 1981) et moins d'hospitalisations en cas de dépression (Lebeau et al., 1979). Enfin, s'il est mieux accepté socialement qu'elles consultent, elles ne manifestent pas plus de symptômes que les hommes mais ces symptômes sont différents (Tousignant, 1980).

L'Enquête Santé Québec 1987 confirme ces différences hommesfemmes. Ces dernières déclarent plus de problèmes de santé physique et mentale que les hommes et utilisent plus souvent des services et médicaments. Les hommes ont encore trois fois plus de problèmes reliés à l'alcool que les femmes; toutefois, ces dernières ont des problèmes psychologiques sévères deux fois plus souvent qu'eux et leur score de détresse psychologique est significativement plus élevé que celui de ces derniers (Émond et Guyon, 1988).

\section{Des facteurs explicatifs}

Le type de socialisation des filles et les rôles féminins pourraient bien expliquer cette réalité de même que l'impact négatif de doubles standards de santé mentale chez des intervenants surtout masculins. Les auteures féministes reconnaissent en effet des causes socioculturelles aux problèmes des femmes. Ces causes sont la socialisation qui conduit les femmes à adopter des comportements passifs et dépendants, ainsi que les rôles limités, peu valorisés et discontinus qui sont attendus d'elles. Ces facteurs ne favorisent pas le plein développement de leur potentiel et conduisent également à des problèmes de détresse psychologique (Belotti, 1974; Corbeil et al., 1983; Gove et Tudor, 1973; Rawlings et Carter, 1977; Russell, 1984; Sturdivant, 1980; Wyckoff, 1977a).

L'analyse critique des modèles traditionnels d'intervention a mis en lumière l'existence de standards de santé mentale différents pour les hommes et les femmes, les stéréotypes sexistes présents chez les intervenants et leurs impacts négatifs (Broverman et al., 1970; Corbeil et al., 1983; Guyon et al., 1981; Sturdivant, 1980). Par ailleurs, les caractéristiques féminines traditionnelles sont associées à des problèmes dépressifs, à une pauvre estime de soi, à des problèmes d'affirmation ou encore à des difficultés d'adaptation sociale alors que les personnalités androgynes sont mieux équilibrées et adaptées socialement (Bem, 1974 : voir DeKoninck et al., 1981; Spence et al., s.d. : voir Beaudry, 1981; Serbin et al., 1979 : voir DeKoninck, 1981; Jakubowski, 1977a).

\section{L'intervention féministe}

L'intervention féministe présente une alternative d'aide qui favorise le développement des femmes en palliant aux lacunes de leur 
éducation et aux possibilités d'autoréalisation qui leur sont traditionnellement offertes. C'est une intervention " auprès des femmes, par des femmes, pour des femmes " (Russell, 1984 : 3). C'est un processus d'aide qui poursuit une double cible : une action individuelle et immédiate sur la personne et un changement social à plus long terme. Elle vise à promouvoir l'indépendance, I'autonomie et la pleine réalisation de soi (Corbeil et al., 1983; Rawlings et Carter, 1977; Russell, 1984; Sturdivant, 1980).

La philosophie développée à partir d'une analyse critique de la situation des femmes et des premiers groupes de prise de conscience a conduit à diverses stratégies : la prise de conscience (Brodsky, 1977; Kirsh, 1974), l'entraînement à l'affirmation de soi (Jakubowski, 1977a), une intervention en groupe (Corbeil et al., 1983; Harrison, 1983; Jakubowski, 1977b; Rawlings et Carter, 1977 : 62-63; Wyckoff, 1980) et une vision positive de la cliente dans une relation égalitaire (Corbeil et al., 1983; Legault, 1980; Rawlings et Carter, 1977; Russell, 1984; Sturdivant, 1980; Wyckoff, 1980). À ceci se sont ajoutées des techniques propres à l'intervention féministe : évaluation positive des femmes, analyse sociale, encouragement à des comportements androgynes, rétroaction aux comportements et autorévélation (Russell, 1984).

\section{L'intervention de groupe}

La revue des principaux modèles d'intervention de groupe en service social et dans des domaines connexes a conduit à des emprunts à l'approche de groupe structuré (Drum et Knott : voir Papell et Rothman, 1983; Middleman, 1981), avec un cadre de référence qui s'appuie sur le modèle du courant central (Papell et Rothman, 1983) et sur la typologie des groupes (Darveau-Fournier et Home, 1983) pour l'articulation du programme.

Même si l'intervention féministe privilégie un modèle de fonctionnement où une grande place est accordée à la cliente, l'approche de groupe structuré offre une contribution particulière au développement d'un programme où les thèmes abordés rejoignent le vécu et les besoins communs des femmes dans une perspective féministe (analyse sociale, apprentissage de comportements nouveaux et androgynes). Il offre à des femmes, plus souvent isolées les unes des autres, la possibilité de vivre, dans un contexte délimité dans le temps, ce que Middleman appelle "I'expérience de groupe construit ». (1981 : 189). La place accordée à I'enseignement et à l'apprentissage permet aux femmes d'expérimenter des comportements nouveaux dans un contexte favorable, ce qui rejoint la perspective de resocialisation de l'intervention féministe, des études confirmant la 
pertinence de groupes composés uniquement de femmes (Home, 1987).

Le modèle du courant central sert de cadre de référence à l'élaboration du programme par l'accent mis sur les caractéristiques communes aux groupes de service social : objectif commun à tous les membres, processus de groupe, changement individuel et social, expériences et activités variées, participantes actives et capables de s'engager dans un changement, aide mutuelle et authenticité des animatrices dont les rôles sont variés.

La typologie des groupes, basée sur l'objectif principal du programme (et plus souvent sur la présence d'objectifs primaires et secondaires) permet d'articuler les rôles des animatrices, les attentes envers les participantes et le contenu des rencontres, en fonction du développement du groupe et selon que les activités sont orientées vers les objectifs principaux de changement personnel ou vers les objectifs secondaires de développement, comme on le verra dans le tableau 2 ci-après.

\section{Le programme}

L'évaluation porte sur les effets d'un groupe de changement personnel dont le programme (voir tableau 1) combine des activités de prise de conscience et d'apprentissage de l'affirmation de soi, dans un groupe de femmes, en vue de favoriser un changement selon une adaptation du modèle de Wyckoff (1980). C'est une intervention féministe selon la définition de Russell (1984) : auprès d'une clientèle uniquement féminine, exécutée par des animatrices et pour des femmes afin de répondre à leurs besoins spécifiques et de favoriser une meilleure utilisation de leur potentiel comme femmes et comme personnes. Il s'agit d'un groupe fermé qui comprend onze rencontres plus une rencontre de relance.

Le programme vise plus particulièrement une amélioration de l'estime de soi et de l'affirmation de soi des participantes comme objectifs primaires, et la socialisation et l'entraide comme objectifs secondaires. Influencé par l'approche de groupe structuré, il s'articule autour des thèmes communs aux besoins et aux problèmes des femmes : éducation et socialisation, rôles, santé, estime, affirmation, corps et sexualité, solitude et loisirs. Les thèmes abordés tiennent compte des étapes de développement du groupe et du rythme des membres; on passe ainsi de thèmes plus généraux à des thèmes plus personnels.

Le programme conserve aussi une certaine flexibilité, entre autres par un retour sur la semaine et la tâche. Chaque rencontre se 
TABLEAU 1

Modèle d'intervention

\begin{tabular}{|c|c|c|c|c|c|}
\hline CONSCIENCE & + & ACTION & + & CONTACT & POUVOIR \\
\hline \multicolumn{6}{|c|}{ Equation qui résume le programme : } \\
\hline $\begin{array}{l}\text { Prise de conscience } \\
\text { de soi } \\
\text { comme personne } \\
\text { comme femme }\end{array}$ & & $\begin{array}{l}\text { Apprentissage de l'af- } \\
\text { firmation et réalisation } \\
\text { d'un objectif person- } \\
\text { nel }\end{array}$ & + & $\begin{array}{l}\text { Support entraide par- } \\
\text { tage en groupe }\end{array}$ & $\begin{array}{l}\longrightarrow \text { Changements dans les } \\
\text { attitudes et compor- } \\
\text { tements }\end{array}$ \\
\hline
\end{tabular}


TABLEAU 2

Le programme

\begin{tabular}{|c|c|c|c|c|c|}
\hline $\begin{array}{l}\text { Objectif } \\
\text { général }\end{array}$ & $\begin{array}{c}\text { Objectifs } \\
\text { spécifiques }\end{array}$ & $\begin{array}{c}\text { Objectifs } \\
\text { particularisés }\end{array}$ & Thèmes & $\begin{array}{c}\text { Activités/ } \\
\text { techniques }\end{array}$ & Rôles \\
\hline $\begin{array}{l}\text { Produire } \\
\text { un chan- } \\
\text { gement } \\
\text { personnel } \\
\text { au niveau } \\
\text { des atti- } \\
\text { tudes des } \\
\text { femmes et } \\
\text { de leurs } \\
\text { comporte- } \\
\text { ments : } \\
\text { Les amener } \\
\text { à mieux } \\
\text { utiliser et } \\
\text { développer }\end{array}$ & $\begin{array}{l}\text { Améliorer l'es- } \\
\text { time de soi : } \\
\text { reconnaissance } \\
\text { de sa valeur per- } \\
\text { sonnelle } \\
\text { - comme } \\
\text { femme } \\
\text { - comme per- } \\
\quad \text { sonne }\end{array}$ & $\begin{array}{l}\text { Prendre conscience } \\
\text { de l'impact de la } \\
\text { socialisation, des } \\
\text { rôles imposés et } \\
\text { des stéréotypes sur } \\
\text { l'image de soi et la } \\
\text { santé mentale. } \\
\text { Apprendre à iden- } \\
\text { tifier ses sen- } \\
\text { timents, à nommer } \\
\text { un vécu et à recon- } \\
\text { naître sa valeur per- } \\
\text { sonnelle. }\end{array}$ & $\begin{array}{l}\text { Socialisation et } \\
\text { rôles } \\
\text { La femme et son } \\
\text { corps } \\
\text { Santé }\end{array}$ & $\begin{array}{l}\text { Analyse sociale } \\
\text { Discussion } \\
\text { "Remue-ménin- } \\
\text { ges " } \\
\text { Information } \\
\text { Autorévélation } \\
\\
\text { Mises en situa- } \\
\text { tion } \\
\text { Tâches } \\
\text { Liens entre in- } \\
\text { formation et } \\
\text { vécu individuel } \\
\text { et de groupe }\end{array}$ & \multirow{2}{*}{$\begin{array}{l}\text { Thérapeutes } \\
\\
\text { Enseignantes }\end{array}$} \\
\hline $\begin{array}{l}\text { tiel pour } \\
\text { résoudre } \\
\text { leurs pro- } \\
\text { blèmes. }\end{array}$ & $\begin{array}{l}\text { Augmenter la } \\
\text { capacité de s'af- } \\
\text { firmer: }\end{array}$ & $\begin{array}{l}\text { Apprendre à iden- } \\
\text { tifier ses difficultés } \\
\text { d'affirmation. }\end{array}$ & $\begin{array}{l}\text { Affirmation de } \\
\text { soi }\end{array}$ & $\begin{array}{l}\text { Information } \\
\text { Discussion } \\
\text { Exercices d'affir- } \\
\text { mation }\end{array}$ & \\
\hline
\end{tabular}




\begin{tabular}{|c|c|c|c|c|}
\hline $\begin{array}{l}\text { meilleure utilisa- } \\
\text { tion de ses } \\
\text { capacités per- } \\
\text { sonnelles dans } \\
\text { les rapports in- } \\
\text { terpersonnels et } \\
\text { dans les tâches }\end{array}$ & $\begin{array}{l}\text { Apprendre à distin- } \\
\text { guer entre pas- } \\
\text { sivité, affirmation et } \\
\text { agression. } \\
\text { Apprendre des } \\
\text { habiletés nouvelles } \\
\text { et développer des } \\
\text { comportements an- } \\
\text { drogynes. } \\
\text { Réaliser un objectif } \\
\text { individuel. }\end{array}$ & $\begin{array}{l}\text { Affirmation de } \\
\text { soi } \\
\text { Relaxation } \\
\text { Affirmation de } \\
\text { soi } \\
\text { La femme et son } \\
\text { corps } \\
\text { Rencontre sur } \\
\text { objectif et bilan }\end{array}$ & $\begin{array}{l}\text { Mises en situa- } \\
\text { tion } \\
\text { "Modeling" } \\
\text { Partage de l'in- } \\
\text { formation et du } \\
\text { savoir } \\
\text { Documents } \\
\\
\text { Tâches } \\
\text { Feuille de route }\end{array}$ & Facilitatrices \\
\hline $\begin{array}{l}\text { Favoriser la } \\
\text { socialisation }\end{array}$ & $\begin{array}{l}\text { Augmenter le sen- } \\
\text { timent de com- } \\
\text { pétence sociale. } \\
\text { Découvrir des } \\
\text { moyens de vaincre } \\
\text { l'isolement et d'ap- } \\
\text { privoiser la } \\
\text { solitude. }\end{array}$ & $\begin{array}{l}\text { Toutes les ren- } \\
\text { contres } \\
\text { Socialisation et } \\
\text { rôles } \\
\text { Solitude et } \\
\text { loisirs }\end{array}$ & $\begin{array}{l}\text { Stimulation des } \\
\text { contacts dans le } \\
\text { groupe } \\
\text { Lien, généralisa- } \\
\text { tions } \\
\text { Travail en sous- } \\
\text { groupes } \\
\text { Analyse sociale }\end{array}$ & Coanimatrices \\
\hline $\begin{array}{l}\text { Développer l'en- } \\
\text { traide et le sup- } \\
\text { port }\end{array}$ & $\begin{array}{l}\text { Encourager et } \\
\text { stimuler les mani- } \\
\text { festations d'en- } \\
\text { traide. } \\
\text { Favoriser la } \\
\text { découverte de } \\
\text { similitudes et dif- } \\
\text { férences. }\end{array}$ & Tous les thèmes & $\begin{array}{l}\text { Partage du } \\
\text { vécu/liens } \\
\text { Valorisation des } \\
\text { comportements } \\
\text { d'entraide et de } \\
\text { support }\end{array}$ & \\
\hline
\end{tabular}


déroule selon un plan semblable ce qui assure une certaine sécurité aux membres : présentation du programme de la soirée, exercice de relaxation, retour sur la semaine et sur la tâche, thème de la rencontre, pause, évaluation de la rencontre et tâche de la semaine.

Des activités variées favorisent une implication active des femmes malgré leur dépendance initiale, à travers une relation égalitaire, où les animatrices jouent, en coanimation, des rôles de thérapeutes, enseignantes et facilitatrices. Le tableau 2 résume le programme.

\section{La méthodologie de la recherche}

Cette recherche a comme objectif l'évaluation des effets du programme. Celui-ci répond aux critères d'évaluabilité : un programme clairement articulé, des objectifs et effets attendus clairement précisés ainsi qu'un lien logique entre le programme et les objectifs et effets attendus (Rutman, 1982). Le programme s'est déroulé conformément à la description qui en est donnée (voir tableau 2); on tient donc pour acquis que c'est le même programme qui a été appliqué aux trois groupes successifs sur lesquels porte l'évaluation.

Les objectifs d'évaluation portent sur trois dimensions de l'atteinte des deux objectifs principaux du programme : I'amélioration de l'estime de soi et de l'affirmation de soi et la diminution de l'état dépressif (tableau 3).

L'hypothèse principale est que le programme (variable indépendante) contribue à améliorer l'estime de soi, I'affirmation de soi et l'état dépressif des participantes (variables dépendantes). Ces variables sont définies de façon opérationnelle et mesurées à l'aide de grilles d'auto-évaluation : I'Inventaire d'estime de soi (Hudson, 1982; Boisvert et Comeau, 1985), I'Inventaire d'affirmation de soi (Gambrill et Richey, 1975), et l'Auto-évaluation de l'état dépressif de Beck (Gauthier et al., 1982). D'autres variables décrivent les caractéristiques sociodémographiques, familiales et économiques des participantes, la nature de leurs problèmes (entre autres un diagnostic de névrose ou de psychose), les autres formes d'aide auxquelles elles ont recours, et leur participation aux rencontres; certaines sont aussi considérées comme variables intermédiaires.

Le devis semi-expérimental de recherche comprend un groupe expérimental mesuré avant le programme, immédiatement après les rencontres et trois mois plus tard; il comprend aussi deux groupes de contrôle dont l'un mesuré avant seulement, et l'autre, trois mois plus tard. Malgré ses limites, ce devis permet de tenir compte du contexte clinique, des contraintes de temps et de la disponibilité de la clientèle 


\section{TABLEAU 3}

\section{Cadre d'évaluation de l'étude}

\begin{tabular}{|c|c|c|}
\hline Les clientes & Le programme & Les effets \\
\hline $\begin{array}{l}\text { Femmes qui consultent en } \\
\text { psychiatrie pour divers } \\
\text { problèmes de santé men- } \\
\text { tale }\end{array}$ & $\begin{array}{l}\text { Service social de groupe } \\
\text { avec approche féministe } \\
\text { à court terme }\end{array}$ & $\begin{array}{l}\text { - Améliorer l'estime de soi } \\
\text { - Améliorer I'affirmation de soi : } \\
\text { - diminuer le malaise } \\
\text { - augmenter les comportements } \\
\text { affirmatifs } \\
\text { - Diminuer l'état dépressif }\end{array}$ \\
\hline
\end{tabular}


pour la réalisation du programme, tout en offrant une base de comparaison.

La vérification des hypothèses est effectuée avec le test $t$ des moyennes ${ }^{2}$. Des distributions de fréquences et des statistiques descriptives sont aussi utilisées, le traitement statistique étant effectué avec le logiciel Stat-Pac, version 6.0 (Walonick, 1982).

\section{Les caractéristiques des participantes}

Les participantes de l'étude se répartissent en trois groupes, un groupe expérimental de 9 femmes et deux groupes de contrôle de 7 et 10 femmes. Elles ont entre 28 et 53 ans avec une moyenne de 40 ans. Une moitié d'entre elles sont mariées et près des deux tiers ont terminé leur cours secondaire. La majorité ont des enfants et $60 \%$ n'occupent aucun emploi hors du foyer : leurs rôles sont donc limités et traditionnels. Elles se partagent également entre les niveaux inférieur et supérieur de statuts socio-économiques.

Les participantes décrivent leurs problèmes en termes d'estime de soi, de symptômes psychiatriques et de solitude et ceux-ci sont diagnostiqués névrotiques dans la moitié des cas et psychotiques dans l'autre. La majorité ont déjà eu recours à d'autres formes d'aide (consultation, hospitalisation, médication), les ont cessées (sauf la médication) pendant le programme et les ont poursuivies par la suite. La participation aux rencontres et le taux de satisfaction sont élevés.

Les trois groupes sont assez semblables. Les femmes du groupe expérimental sont cependant plus âgées, demeurent surtout au foyer et leur statut socio-économique est plus faible; leurs problèmes paraissent plus lourds et elles ont été moins assidues aux rencontres. En ce qui concerne l'estime de soi, l'affirmation de soi et l'état dépressif, elles ne diffèrent pas dans leur ensemble des femmes des groupes de contrôle, ni avant les rencontres ni au moment de la relance, même si l'écart est grand entre les sujets d'un même groupe.

\section{Les effets du programme}

\section{L'estime de soi}

L'ensemble des participantes n'ont pas amélioré leur estime d'elles-mêmes de façon statistiquement significative, tant immédiatement après les rencontres que trois mois plus tard. Toutefois, en présence d'un diagnostic de névrose, on observe une amélioration de l'estime de soi immédiatement après le programme et celle-ci se 
maintient encore trois mois plus tard. On observe aussi une amélioration dans le groupe des participantes plus jeunes ( 40 ans et moins) immédiatement après le programme mais non trois mois plus tard; à ce moment on observe cependant une amélioration chez les femmes n'ayant pas été hospitalisées antérieurement.

Ces résultats sont aussi confirmés par l'analyse cas par cas qui révèle une nette amélioration chez les femmes névrotiques présentant des problèmes d'estime importants avant leur participation, alors que celles qui n'avaient pas de problèmes d'estime de soi se sont maintenues au même point avant et après le programme. Au contraire, le programme n'a pas amélioré l'estime de soi (à une exception près révélée par l'analyse cas par cas) lorsqu'on est en présence d'un diagnostic de psychose.

Le programme de groupe avec approche féministe évalué a donc des effets bénéfiques pour une moitié des participantes : la nature des difficultés rencontrées (diagnostic) et leur gravité (hospitalisation) semblent être des facteurs qui aident à prédire les succès du programme à améliorer l'estime de soi. Ces résultats diffèrent cependant d'une étude qui rapporte une amélioration de l'estime de soi auprès de femmes présentant des problèmes sévères (dont une majorité de psychoses) et étiquetées chroniques par leurs thérapeutes (Alyn et Becker, 1984).

\section{L'affirmation de soi}

On observe aussi une amélioration de l'affirmation de soi d'une partie des femmes en présence de certaines caractéristiques personnelles ou situationnelles; I'amélioration observée se manifeste soit par un moindre malaise, soit par des comportements affirmatifs plus nombreux, selon les cas. C'est au moment de la relance seulement que l'ensemble des participantes manifestent une diminution sensible de leur niveau de malaise à s'affirmer, alors qu'on percevait une tendance en ce sens à la fin des rencontres. Quant aux comportements affirmatifs, on n'observe qu'une tendance à plus d'affirmation trois mois après la fin des rencontres.

Dans le cas de la diminution significative du malaise observée au moment de la relance, cette amélioration se maintient seulement en présence du recours à une autre forme d'aide thérapeutique après le programme.

Les quelques professionnels référants rapportent cependant que certaines femmes, qui sont retournées les consulter après leur participation au programme, manifestaient une attitude plus active et plus déterminée dans la définition de l'aide attendue. Si le programme avait produit un tel effet, ce serait aussi à considérer. 
La nature du problème présenté semble aussi influencer l'amélioration de l'affirmation de soi, même si c'est moins évident que dans le cas de l'estime de soi. Les participantes ayant des problèmes névrotiques ressentent moins de malaise et se comportent plus affirmativement après le programme, même si seule une diminution du malaise à la relance est statistiquement confirmée. À l'inverse, on n'observe aucune amélioration de l'affirmation de soi (ni du malaise, ni des comportements affirmatifs) en présence $d^{\prime} u n$ diagnostic de difficultés psychotiques.

Dans leur ensemble, les effets observés se rapprochent de ceux obtenus par Sprei-Ott (1976). Celle-ci a en effet noté une amélioration de l'affirmation de soi auprès de femmes moyennement déprimées, suite à deux types de programme de groupe, l'un axé sur l'affirmation de soi et l'autre sur la prise de conscience. Comme sa population ne comprenait que des femmes déprimées, excluait celles ayant des tendances suicidaires et qui faisaient usage d'antidépresseurs, ces femmes se rapprochaient probalement, au niveau de la gravité des problèmes, des femmes névrotiques de notre étude (où nous retrouvons plusieurs problèmes dépressifs) pour lesquelles une amélioration de l'affirmation de soi est plus sensible.

\section{L'état dépressif}

Au niveau de l'état dépressif, on observe une diminution qui s'approche beaucoup du seuil de signification retenu immédiatement après le programme et l'amélioration est significative lors de la relance, trois mois plus tard. Encore ici la nature du problème semble un facteur déterminant du succès du programme. En effet, les femmes ayant un diagnostic de problèmes névrotiques diminuent sènsiblement leur état dépressif (près du seuil de signification après le programme et de manière significative à la relance). Celles dont les difficultés sont de nature psychotique ont une amélioration trop faible pour qu'on puisse y porter attention.

Immédiatement après le programme, la diminution de l'état dépressif est associée au fait que la participante juge que le programme lui a été beaucoup utile. On observe également, à ce moment, une amélioration chez les participantes dont les problèmes existent depuis plus longtemps.

À la relance, le changement est associé à un plus grand nombre de présences, ce qui tend à démontrer que le programme produit effectivement les effets qu'on lui attribue. Cependant, on doit aussi retenir que les femmes qui ont poursuivi d'autres démarches d'aide et qui ont continué à prendre une médication sont moins déprimées, alors que les effets du programme sont moins visibles chez les autres. 
Ces résultats vont dans le même sens que ceux de Sprei-Ott (1976). Toutefois, même si on a observé une diminution de l'état dépressif, immédiatement après le programme et trois mois plus tard, chez les femmes ayant des problèmes névrotiques, et qu'elles se situent alors en deçà du seuil de dépression, elles demeurent cependant encore plus déprimées que la moyenne des femmes québécoises (Gauthier et al., 1982).

\section{Commentaires et conclusions}

Même si certaines participantes se sont améliorées suite aux rencontres, la recherche ne supporte pas les intuitions cliniques initiales; l'hypothèse générale de la recherche à l'effet que le programme améliore l'estime de soi, l'affirmation de soi et l'état dépressif doit donc être rejetée. Pour l'ensemble des participantes, I'hypothèse d'une amélioration ne se vérifie que trois mois après la fin des rencontres, et uniquement sous deux aspects : diminution de l'état dépressif et du malaise face à l'affirmation de soi.

Comme on ne peut contrôler l'ensemble des événements survenus après le programme et que les changements observés se maintiennent en présence du recours à une autre forme d'aide suite aux rencontres et à l'usage d'une médication (souvent des antidépresseurs), on ne peut attribuer les changements seulement au programme. Tout au plus, peut-on penser qu'il y a amélioration lorsque les participantes peuvent compter sur un autre appui en plus de leur participation aux rencontres.

Le programme paraît aussi plus utile en présence de troubles névrotiques. Ces femmes améliorent leur estime d'elles-mêmes de façon significative immédiatement après le programme et cela se maintient trois mois plus tard. Elles ressentent significativement moins de malaise et leurs comportements affirmatifs sont plus nombreux après trois mois. Enfin, leur état dépressif diminue immédiatement après le programme et de façon statistiquement significative à la relance. Les améliorations observées individuellement chez les participantes au niveau de l'estime de soi, et la comparaison avec les changements dans l'affirmation de soi et l'état dépressif, confirment aussi un changement plus continu en présence d'un diagnostic de troubles névrotiques.

À l'inverse, en présence d'un diagnostic de troubles psychotiques, on n'observe aucune amélioration de l'estime de soi, de l'affirmation de soi, ni de l'état dépressif, après les rencontres et trois mois plus tard. II n'est pas possible de relier les changements 
observés à d'autres caractéristiques des participantes, à leurs problèmes ou à leur assiduité aux rencontres.

Enfin les changements observés apparaissent à des moments différents. Au niveau de l'estime de soi, l'amélioration, en présence d'un diagnostic de troubles névrotiques, est plus importante immédiatement après les rencontres et diminue après trois mois. L'inverse se produit pour les autres mesures : en ce qui concerne le malaise face à l'affirmation, les comportements affirmatifs et l'état dépressif, les améliorations sont faibles immédiatement après les rencontres et s'amplifient après trois mois.

Comment expliquer l'apparition plus ou moins rapide de certaines améliorations? Sont-elles reliées à des éléments précis du programme ou à certaines caractéristiques des participantes et de leur situation ? Le programme est-il réellement utile aux seules femmes névrotiques ? Une approche cognitive et à court terme est-elle moins indiquée en présence de problèmes psychotiques ? Ces femmes tirent-elles d'autres avantages de leur participation au programme que les cibles d'évaluation retenues ne font pas ressortir? La recherche actuelle ne permet pas de répondre à ces questions.

Ces résultats et les questions qu'ils suscitent doivent enfin être situés dans le contexte des limites de l'étude, dont le petit nombre de sujets et leurs différences individuelles avant le programme, le devis quasi expérimental qui ne permet pas d'éliminer certaines autres explications possibles et, enfin, les erreurs de mesure liées aux échelles d'auto-évaluation. À ce dernier niveau, il faut en effet noter l'anxiété soulevée surtout par la mesure avant le programme. De plus, les participantes présentant des troubles psychotiques ont semblé minimiser les problèmes selon les impressions cliniques des animatrices.

\section{Implications pour la pratique et la recherche}

Même si les résultats observés ne permettent pas de conclure avec évidence à l'utilité d'une intervention de groupe avec approche féministe pour l'ensemble des femmes qui consultent dans une clinique externe de psychiatrie, les améliorations partielles observées et les limites mêmes de l'étude nous amènent à suggérer la poursuite de cette forme d'intervention auprès des femmes, le développement d'un progrmme complémentaire et la poursuite de la recherche.

Dans l'état actuel de nos connaissances, il apparaît pertinent que ce programme puisse être offert aux femmes comme complément à d'autre formes d'aide, dès leur demande initiale de services ou encore comme dernière étape vers un fonctionnement autonome, 
selon la nature de leurs difficultés ou le jugement clinique des référants. Plusieurs participantes ont en effet trouvé les rencontres normalisantes et valorisantes.

Les améliorations partielles observées semblent aussi confirmer les demandes de certaines d'entre elles pour un programme complémentaire. Un tel programme pourrait être moins structuré et impliquer les participantes dans la définition de ses objectifs et de son contenu afin d'éviter de les maintenir dans la dépendance et de favoriser l'intégration des changements. L'accent devrait être mis sur le développement de l'entraide et la socialisation des participantes.

La présente recherche n'est qu'un premier pas vers une meilleure connaissance des effets d'un programme de groupe avec approche féministe en santé mentale. De telles recherches devraient être répétées sur une plus grande échelle afin de pouvoir comparer les résultats selon les caractéristiques particulières des participantes ou la nature des problèmes présentés.

La recherche d'effets du programme, autres que ceux mesurés, particulièrement chez les femmes psychotiques, et l'identification de facteurs du programme qui favorisent l'apparition plus ou moins rapide des améliorations et leur maintien, seraient d'autres pistes à explorer. Ainsi pourrait-on en arriver à identifier des modèles de changement associés à certaines caractéristiques, modèles semblables à ceux identifiés par Home (1980) auprès de groupes de conscientisation.

\section{Notes}

1 Brousseau-Gingras, Michèle, Effets d'un programme d'intervention de groupe avec approche féministe sur l'estime de soi, l'affirmation de soi et l'état dépressif, Mémoire de maîtrise en service social, Université Laval, 1988, $174 \mathrm{p}$.

${ }^{2}$ Le test t est une mesure statistique qui convient à des échantillons aussi petits que les groupes à l'étude $(\mathrm{N}<30)$; il permet de comparer les moyennes d'un même groupe de sujets avant et après le programme et les moyennes de deux groupes entre eux.

\section{Références}

AlyN, Jody H. et Lee A. BeCKer (1984). " Feminist Therapy With Chronically And Profoundly Disturbed Women", Journal of Counseling Psychology, vol. 31, no $2: 202-208$.

Beaudry, Madeleine (1981). "Les femmes et les difficultés d'affirmation de

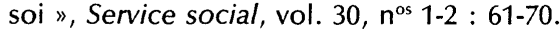

Belottı, E.G. (1974). Du côté des petites filles. Paris : Éditions des femmes. 
BOISVERT, Jean-Marie et Suzanne COMEAU (1985). Un ensemble de mesures cliniques, traduit de W.W. Hudson, inédit.

BRODSkY, A.M. (1977). "Therapeutic Aspects of Consciousness Raising Groups » : 300-310, dans E.I. Rawlings et D.K. Carter (éd.), Psychotherapy For Women : Treatment Toward Equality. Springfield : Thomas.

Broverman, I.K. et al. (1970). "Sex-Role Stereotypes And Clinical Judgements Of Mental Health ", Journal Of Consulting Clinical Psychology, vol. 34, $n^{\circ} 1: 1-7$.

Chesler, Phylis (1979). Les femmes et la folie. Paris : Payot.

COOPERSTOCK, R. (1980). "Problèmes particuliers liés à l'usage de psychotropes chez les femmes", Santé mentale au Canada, vol. 28, n 2.

CORBElL, C. et al. (1983). L'intervention féministe, l'alternative des femmes au sexisme en thérapie. Montréal : Éditions coopératives Albert Saint-Martin.

DARVEAU-FOURNIER, Lise et Alice HOME (1983). " Les groupes de service social à Québec : recherche des rapports entre théorie et pratique ", Service social, vol. 32, $\mathrm{n}^{\text {os }} 1-2: 129-156$.

DeKoninck, M., F. Saillant et L. Dunnigan (1981). Essai sur la santé des femmes. Québec, Conseil du statut de la femme.

ÉMOND, Aline et Louise GuYON (1988). La santé des Québécois et des Québécoises: Faits saillants, Enquête Santé Québec 1987. Québec, Gouvernement du Québec, Ministère de la Santé et des Services sociaux.

GAMBRILL, E.D. et C.A. RiChey (1975). "An Assertion Inventory for Use in Assessment and Research ", Behavior Therapy, $\mathrm{n}^{\circ} 6: 550-560$.

Gauthier, J., F. Thériault et C. Morin (1982). "Adaptation française d'une mesure d'auto-évaluation de l'intensité de la dépression (Beck) ", Revue Québécoise de psychologie, vol. 3, $\mathrm{n}^{\circ} 2$ : 13-27.

Gove, W.R. et J. TUdor (1973). "Adult Sex Roles and Mental Illness", American Journal of Sociology, $\mathrm{n}^{\circ} 78: 812-835$.

GUYON, Louise, Roxane SIMARD et Louise NADEAU (1981). "Va te faire soigner, t'es malade!". [Montréal] : Stanké.

HARRISON, S.W. (1983). "Thérapie et conscientisation dans les groupes thérapeutiques pour femmes", Actualités en analyse transactionnelle, vol. 7, $\mathrm{n}^{\circ} 27: 129-134$.

HOME, Alice (1980). "Étude exploratoire de quatre types de changements survenus chez les membres de groupes féminins de conscientisation ", Service social, vol. $29, \mathrm{n}^{\text {os }} 1-2: 152-182$.

Home, Alice (1987). Les femmes, le sexisme et les petits groupes : réflexions sur la théorie des groupes. Les cahiers de recherche du GREMF : $\mathrm{n}^{\circ} 11$. Québec, Université Laval.

Hudson, Walter W. (1982). The Clinical Measurement Package : a Field Manual. Illinois : Dorsey Press.

JAKUBOWSKI, P.A. (1977a). "Assertive Behaviour and Clinical Problems of Women " : 147-167, dans E.I. Rawlings et D.K. Carter (éd.), Psychotherapy for Women : Treatment Toward Equality. Springfield : Thomas.

JAKUBOWSKI, P.A. (1977b). "Self-Assertion Training Procedures for Women »: 168-193, dans E.I. Rawlings et D.K. Carter (éd.), Psychotherapy for Women : Treatment Toward Equality. Springfield : Thomas.

KIRSH, B. (1974). "Consciousness-raising Groups as Therapy for Women ": 326-354, dans V. Franks et V. Burtle (éd.), Women in Therapy. New York : Brunner/Mazel. 
LeBEAU, A. et al. (1979). "Les urgences psychiatriques dans la région de Montréal Métropolitain. Quelques différences entre les hommes et les femmes", Santé mentale au Québec, vol. 4, n $2: 25-52$.

LeGault, Gisèle (1980). " D'une approche féministe auprès des femmes en service social », Intervention, $\mathrm{n}^{\circ} 57: 3-9$.

MidDleman, Ruth R. (1981). "The Pursuit of Competence through Involvement in Structured Groups»: 185-210, dans A.N. Maluccio (éd.), Promoting Competence in Clients. New York : Free Press.

PAPELL, Catherine et Beulah Rothman (1983). "Le modèle du courant central du service social des groupes en parallèle avec la psychothérapie et l'approche de groupe structuré ", Service social, vol. 32, $\mathrm{n}^{\text {os }}$ 1-2 : 11-32.

Rawlings, E.I. et Dianne K. Carter (1977). Psychotherapy for Women: Treatment Toward Equality. Springfield : Thomas.

RusSeLL, Mary N. (1984). Skills in Counselling Women : The Feminist Approach. Springfield : Thomas.

RUTMAN, Leonard (1982). "Planification d'une étude évaluative " : 23-46, dans R. Lecomte et L. Rutman, Introduction aux méthodes de recherche évaluative. Québec: Presses Université Laval.

SPREI-OTT, Judith Ellen (1979). The Use of Assertiveness Training and Consciousness-Raising Groups in the Treatment of Depression in Women. University of Maryland, thèse de doctorat inédite.

Sturdivant, Susan (1980). Les femmes et la psychothérapie, une philosophie féministe du traitement. Bruxelles : Mardaga.

TousignANT, M. et al. (1980). "Les biais reliés au sexe dans les études de santé mentale ", dans Les femmes et la folie, $5^{\mathrm{e}}$ colloque sur la santé mentale, Montréal.

WALONICK, David S. (1986). StatPac - Statistical Analysis Package for the IBM, version 6.0.

WYCKOFF, Hogie (1977a). "Radical Psychiatry for Women » : 370-392, dans E.I. Rawlings et D.K. Carter (éd.), Psychotherapy for Women: Treatment Toward Equality. Springfield : Thomas.

WYCKOFf, Hogie (1980). Solving Problems Together. New York : Grove Press. 